\title{
Analysis of mRNA expression profiles of carotenogenesis and astaxanthin production of Haematococcus pluvialis under exogenous 2, 4-epibrassinolide (EBR)
}

\author{
Zhengquan Gao ${ }^{1, a}$, Chunxiao Meng ${ }^{1, a},{ }^{*}$, Hongzheng Gao ${ }^{1}$, Xiaowen Zhang ${ }^{2}$, Dong $\mathrm{Xu}^{2}$, Yuanfeng Su${ }^{1}$, Yuanyuan \\ Wang ${ }^{1}$, Yuren Zhao ${ }^{1}$ and Naihao $\mathrm{Ye}^{2, *}$ \\ ${ }^{1}$ School of Life Sciences, Shandong University of Technology, Zibo 255049, PR China \\ 2 Yellow Sea Fishery Research Institute, Chinese Academy of Fishery Sciences, Qingdao 266071, PR China
}

\begin{abstract}
The fresh-water green unicellular alga Haematococcus pluvialis is known to accumulate astaxanthin under stress conditions. In the present study, transcriptional expression of eight genes involved in astaxanthin biosynthesis exposed to EBR ( 25 and $50 \mathrm{mg} / \mathrm{L}$ ) was analyzed using qRT-PCR. The results demonstrated that both 25 and $50 \mathrm{mg} / \mathrm{L}$ EBR could increase astaxanthin productivity and the eight carotenogenic genes were up-regulated by EBR with different expression profiles. Moreover, EBR25 induction had a greater influence on the transcriptional expression of ipi-1, ipi-2, crtR-B, lyc and crtO (> 5- fold up-regulation) than on $p s y, p d s, b k t$; EBR50 treatment had a greater effect on the transcriptional expression of $i p i-2, p d s, l y c, c r t \mathrm{R}-\mathrm{B}, b k t$ and $c r$ O than on ipi-1 and $p s y$. Furthermore, astaxanthin biosynthesis under EBR was up-regulated mainly by ipi-1 and psy at the post-transcriptional level, $p d s, l y c, c r t \mathrm{R}-\mathrm{B}, b k t$ and $c r t \mathrm{O}$ at the transcriptional level and ipi-2 at
\end{abstract} both levels.

Key terms: Haematococcus pluvialis, astaxanthin, 2, 4-Epibrassinolide (EBR), carotenoid genes, real-time fluorescence quantitative PCR (qRTPCR).

Abbreviations: SA: salicylic acid; JA: jasmonic acid; BRs: Brassinosteroids; EBR: 2, 4-Epibrassinolide

\section{INTRODUCTION}

Astaxanthin (3,3'-dihydroxy- $\beta, \beta$-carotene-4, $4^{\prime}$-dione), used as a feed additive in aquaculture and dietary supplements, is commercially available either from chemical synthesis or natural resources such as microalgae, yeast and crustacean byproducts (Li et al., 2011). Haematococcus pluvialis, a unicellular green microalga, can produce large amounts of red carotenoid astaxanthin when exposed to stress conditions such as high light, nitrogen starvation, etc. It is commonly suggested that the formation of large amounts of astaxanthin in H. pluvialis is a survival strategy under adverse environmental conditions ( $\mathrm{Li}$ et al., 2008).

Previous research elucidated the pathway of astaxanthin biosynthesis in $H$. pluvialis with specific inhibitors, and most of the involved genes have been cloned (Grünewald et al., 2000. Both higher plants and green algae use isopentenylpyrophosphate (IPP) as the carotenoid precursor and share the same pathway of forming $\beta$-carotene. The following specific steps for astaxanthin biosynthesis in $H$. pluvialis are catalyzed by $\beta$-carotene hydroxylase and $\beta$-carotene oxygenase or $\beta$-carotene ketolase (Figure 1 , modified from Grünewald et al., 2000).

Brassinosteroids (BRs) are plant hormones with significant growth-promoting activity and are involved in multiple developmental processes including the cell cycle and mitosis (Howell et al., 2007), apoptosis (Carange et al., 2011), root and hypocotyl length (Howell et al., 2007; Park. 1998), photosynthesis (Anuradha and Rao 2009), the antioxidant system (Ding et al.,
2009; Ozdemir et al., 2004), seed germination (Anuradha and Rao 2001; Ozdemir et al., 2004; Sharma and Bhardwaj 2007; Ding et al., 2009) and modulation of gene expression (Ding et al., 2009). In addition to a role in development, BRs also affect plant tolerance against various stresses including low and high temperature (Dhaubhadel et al., 1999), drought (Pustovoitova et al., 2001; Li et al., 2012) or water stress (Upret and Murt 2004), heavy metals (Sharma and Bhardwaj 2007; Anuradha and Rao 2009; Choudhary 2011; Sharma et al., 2011), hypoxia (Kang et al., 2009) and salt stress (Ozdemir et al., 2004; Saygideger and Deniz 2008; Dalio et al., 2011; Shahid et al., 2011; Samira et al., 2012). Similar effects are induced by its epimer, 2, 4-epibrassinolide (Bajguz and Czerpak, 1996). BRs have been identified from a green alga Hydrodictyon reticulatum (Chlorophyceae) (Yokota et al., 1987). It was demonstrated that EBR could shorten by $65.9 \%$ the time in which algae cells changed to completely red compared to controls (Gao et al., 2011). However, its exact impact on biosynthesis of astaxanthin in $H$. pluvialis at the molecular level is still unknown. Therefore, studying the effect of BR on astaxanthin accumulation and their relation with the expression of carotenoid genes is necessary. In this study we examined the carotenogenic expression pattern of eight genes (ipi-1, ipi-2, psy, $p d s, l y c, c r t \mathrm{R}-\mathrm{B}, b k t$ and $c r \mathrm{O})$ in combination with astaxanthin accumulation in $H$. pluvialis under EBR $(25$ and $50 \mathrm{mg} / \mathrm{L})$, using real-time PCR and light microscope spectrophotometry. Our results indicated that EBR could increase astaxanthin productivity significantly in $H$. pluvialis and stimulate mRNA expressions of the eight carotenogenic genes, with different expression profiles. 


\section{MATERIAL AND METHODS}

Source of algal strain of $H$. pluvialis and cultivation conditions

The H. pluvialis 712 strain was obtained from the Institute of Oceanology, Chinese Academy of Sciences, and preserved in our laboratory. All samples of $H$. pluvialis were grown in MCM medium (Borowitzka et al., 1991) and cultivated according to Gao et al.,(2012a, 2012b). MCM medium contains the following $\left(\mathrm{mg} \mathrm{L}^{-1}\right): \mathrm{KNO}_{3} 200 ; \mathrm{K}_{2} \mathrm{HPO}_{4}, 20 ; \mathrm{MgSO}_{4} \cdot 7 \mathrm{H}_{2} \mathrm{O}, 100 ; \mathrm{CaCl}_{2}$ $.6 \mathrm{H}_{2} \mathrm{O}$, 80; Vitamin $\mathrm{B}_{12}$, 0.004; EDTA, 0.0198; $\mathrm{FeCI}_{3} .6 \mathrm{H}_{2} \mathrm{O}$, 0.0244. $1 \mathrm{~mL}$ of trace element mix (containing in $\mathrm{mg} \mathrm{L}^{-1}$ : $\mathrm{ZnC1}_{2}$, 4.1; $\mathrm{H}_{3} \mathrm{BO}_{3}, 61 ; \mathrm{CoC1}_{3} \cdot 6 \mathrm{H}_{2} \mathrm{O}, 5.1 ; \mathrm{CuSO}_{4} .5 \mathrm{H}_{2} \mathrm{O}, 6.0$; $\left.\mathrm{MnC1}_{2} \cdot 4 \mathrm{H}_{2} \mathrm{O}, 4.1 ;\left(\mathrm{NH}_{4}\right)_{6} \mathrm{Mo}_{7} \mathrm{O}_{24} \cdot 4 \mathrm{H}_{2} \mathrm{O}, 38.0\right)$ was added and the $\mathrm{pH}$ adjusted to 7.0 after autoclaving.

Inducing H. pluvialis with EBR treatment

Algae solutions in the logarithmic phase were divided into three treatments with three replicates for each treatment: the final concentrations of EBR (Real-Times) were $0 \mathrm{mg} / \mathrm{L}$ (controls), $25 \mathrm{mg} / \mathrm{L}$ EBR (EBR 25) and $50 \mathrm{mg} / \mathrm{L}$ EBR (EBR 50). (EBR was dissolved in a small quantity of ethanol and then compounded with dimethylsulfoxide $10 \mathrm{mg} / \mathrm{mL}$, then it was added into the alga culture solution with the final concentrations). An equal amount of dimethylsulfoxide was added to the controls. All the initial volumes of three controls and 6 treatment samples were $2000 \mathrm{~mL}$ of alga solution cultured in sterile Erlenmeyer flasks. $2 \mathrm{~mL}$ of algal solution each time for controls and treatments was harvested

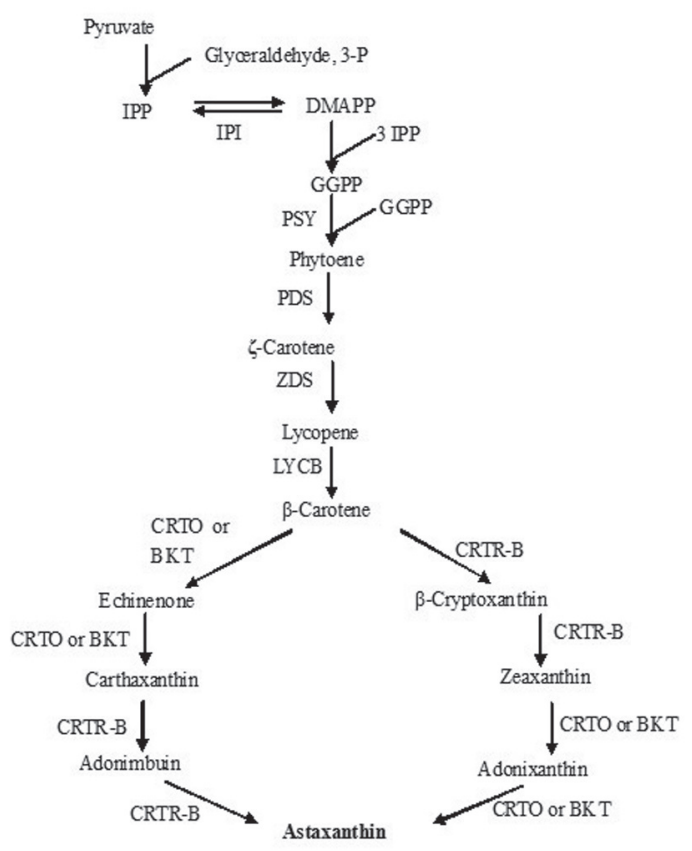

Figure 1: The pathway of astaxanthin biosynthesis in $H$. pluvialis according to Grünewald et al., (2000). Enzyme designation is according to the corresponding gene: CRTL-B, lycopene $\beta$-cyclase; CRTO, $\beta$-carotene oxygenase; CRTR- $B, \beta$-ring hydroxylase; GGPS, geranylgeranyl diphosphate synthase; IPI, isopentenyl diphosphate isomerase; PDS, phytoene desaturase; PSY, phytoene synthase; ZDS, $\zeta$-carotene desaturase. on day $0.5,1,1.5,2,2.5,3,4,6,8,10,12,14,18,22$ for RNA isolation, and $10 \mathrm{~mL}$ of algal solutions each time for controls and treatments was harvested at the beginning of treatment as well as on day $3,6,9,12,15,18$ and 22 for measurement of astaxanthin content. At the end of the experiment, the final volumes of controls and treatments were about $1900 \mathrm{~mL}$.

Microscopic observation and measurement of astaxanthin content

Optical microscopic observation used a Nikon Eclipse 80i microscope (Nikon, Tokyo, Japan) and astaxanthin content was measured with a spectrophotometer (T6 new century, Beijing General Instrument Ltd, China) according to Boussiba and Vonshak, (1991) and Gao et al., (2012a, 2012b). The major absorption peak of dimethylsulfoxide is at ca. $490 \mathrm{~nm}$, and astaxanthin concentration can be calculated according to the formula: $\mathrm{C}(\mathrm{mg} / \mathrm{L})=\left(4.5 \times O D_{490} \times V a\right) / V b$. $V a$ and $V b$ represent the volume of dimethylsulfoxide and microalga sample, respectively. Equal aliquots of culture from each treatment and the control were harvested at different time points and lyophilized. Lyophilized cells were then extracted with dimethylsulfoxide repeatedly until the pellet became colorless. Absorbance of the extracts was read at $490 \mathrm{~nm}$ with a spectrophotometer (T6 new century, Beijing General Instrument Ltd, China). The blank contained dimethylsulfoxide only.

\section{RNA isolation and RT-PCR}

The total RNA of algal samples was extracted using TRIzol Reagent (Invitrogen, USA) according to the user's manual and Gao et al., (2012a, 2012b). The gene-specific primers for eight genes were designed using Primer 3 software and synthesized (Biosune, China) (Table 1). PCR products were quantified continuously with the ABI StepOne Plus RealTime PCR System (Applied Biosystems, USA) using SYBR green fluorescence (Takara) according to the manufacturer's instructions. qRT-PCR analysis of related genes was according to Gao et al., (2012a, 2012b). The actin gene was used as a reference for total RNA. After the PCR program was conducted, the data were analyzed using the comparative $\mathrm{Ct}$ $\left(2^{-\Delta \Delta C T}\right)$ method according to Livak and Schmittgen (2001).

Statistical analysis

The means \pm SD were derived from all data and were statistically analyzed with one-way ANOVA (SPSS 17.0). LSD multiple comparison tests were used to test the differences among groups of different trials. $p$-values of less than 0.05 and 0.01 were considered to be statistically significant and highly significant, respectively.

\section{RESULTS}

Microscopic observation and measurement of astaxanthin content

Microscopic observation showed that algal cells became immobile after 6 hours of application of EBR, which showed existence of the stresses produced by EBR. The initial color changed from green to red on day 2 after application of EBR 25 and EBR 50. On day 3 (data not shown), there was an obvious difference in astaxanthin accumulation between 
controls and treatments. Figure 2 shows the difference among samples under the microscope; A, B, C represent controls and treatments on day 6; D, E, F represent controls and treatments on day $15, \mathrm{G}, \mathrm{H}$, I represent controls and treatments on day 22 , respectively. The calculation of percentages of green, red and bleached algae was carried out microscopically with a hematocytometer. On day 6, albinism of alga cells occurred with percentage of $5.4 \%$ and $11.6 \%$ in EBR25 and EBR50 treatments, respectively. On day 15 , these values frose to $25.5 \%$ and $56.8 \%$ but the rest of the cells became redder. On day 22, more than $55.2 \%$ of the cells lost pigment and bleached, $35.3 \%$ of the cells changed to red in the EBR 25 treatment, and almost $81.1 \%$ of cells bleached and the rest changed completely to red in the EBR 50 treatment (Figure 2). Results from astaxanthin measurements revealed that the fast increasing astaxanthin accumulation began on the $3^{\text {rd }}$ day, and the value climbed quickly continuously and attained its peak on day 9 in the EBR 50 treatment and on day 12 in the EBR 25 treatment. Then the value decreased continuously in both treatments. The astaxanthin contents of 8 time points were $0.073,0.18,0.97$, $1.64,2.26,1.86,1.29$ and $0.88 \mathrm{mg} / \mathrm{L}$ alga culture solution in the EBR25 at the beginning of treatment and on days 3, 6, 9, 12, 15,18 and 22 , respectively. The values were $0.072,0.25,1.39$, $1.98,1.82,1.50,1.07$ and $0.31 \mathrm{mg} / \mathrm{L}$ alga culture solution in the EBR50 treatments at the same eight time points. Moreover, the EBR 25 treatment resulted in a higher astaxanthin production $(2.26 \mathrm{mg} / \mathrm{L}$ alga culture solution) than that of the EBR 50 treatment $(1.98 \mathrm{mg} / \mathrm{L}$ alga culture solution) at the peaks. The astaxanthin content of the controls was $0.084 \mathrm{mg} / \mathrm{L}$ alga culture solution after culture for $22 \mathrm{~d}$ (Figure 3 ). This was in line with our previous results, which indicated that some kinds of exogenous phytohormone such as JA and SA could stimulate H. pluvialis to accumulate astaxanthin (Gao et al., 2012a, 2012b).

\section{Transcriptional patterns of carotenoid genes induced by EBR}

qRT-PCR results showed the initial increased transcriptional levels of ipi-1 in the EBR 25 treatment occurred on day 0.5 with 2.6-fold and the maximum ipi-1 level occurred on day 2 with 5.3-fold, respectively. However, the initial increased and greatest ipi-1 expression occurred on day 2 with 2.3-fold levels in the EBR 50 treatment (Figure 4A). The initial increased and maximum transcription of ipi-2 occurred on day 2 with 6.9 -fold levels in the EBR 25 treatment, then declined sharply until day 22 , whereas the ipi-2 level rose to 3.4 -fold on day 2 and then declined, and reached its highest level on day 12 with 18.8-fold in the EBR 50 treatment (Figure 4B).

The initial increased and maximum transcriptional levels of psy in both treatments occurred on day 2, with 4.5- and 3.1-fold compared to the control, respectively, then declined irregularly until day 22 (Figure 4C). In the EBR 25 treatment, the first peak of $p d s$ level occurred on day 3 (3.1-fold) and the highest peak of $p d s$ level occurred on day 12 (3.6-fold), whereas the initial increased and highest transcriptional level of pds occurred on day 8 (4.3-fold) and day 12 (13.2-fold) in the EBR 25 and 50 treatments, respectively (Figure 4D).

The initial increased transcriptional levels of crtR-B were 2.6- and 3.9-fold on day 0.5 in the EBR 25 treatment. The

TABLE I

Gene-specific primers and annealing temperatures used for qRT-PCR

\begin{tabular}{|c|c|c|c|}
\hline Primer & Primer sequence $\left(5^{\prime}-3^{\prime}\right)$ & annealing temperature $\left({ }^{\circ} \mathrm{C}\right)$ & GenBank ID \\
\hline$p s y \mathrm{~F}$ & CGATACCAGACCTTCGACG & 55 & AF305430 \\
\hline$p s y \mathrm{R}$ & TGCCTTATAGACCACATCCAT & & \\
\hline$p d s \mathrm{~F}$ & ACCACGTCGAAGGAATATCG & 58 & X86783 \\
\hline$p d s \mathrm{R}$ & TCTGTCGGGAACAGCCG & & \\
\hline$l y c \mathrm{~F}$ & TGGAGCTGCTGCTGTCCCT & 61 & AY182008 \\
\hline $\operatorname{lycR}$ & GAAGAAGAGCGTGATGCCGA & & \\
\hline$c r t R-b F$ & ACACCTCGCACTGGACCCT & 62 & AF162276 \\
\hline $\operatorname{crt} R-\mathrm{b} R$ & GTATAGCGTGATGCCCAGCC & & \\
\hline$b k t 2 \mathrm{~F}$ & CAATCTTGTCAGCATTCCGC & 61 & AY603347 \\
\hline$b k t 2 R$ & $\begin{array}{l}\text { CAGGAAGCTCATCACATCAGAT } \\
\text { GCGAGCACGAAATGGACTAC }\end{array}$ & & \\
\hline ipi-1F & GCTGCATCATCTGCCGCA & 61 & AF082325 \\
\hline ipi-1R & AGTACCTGGCGCAAAAGCTG & & \\
\hline$i p i-2 \mathrm{~F}$ & GTTGGCCCGGATGAATAAGA & 62 & AF082326 \\
\hline ipi-2R & ACGTACATGCCCCACAAG & & \\
\hline crtOF & CAGGTCGAAGTGGTAGCAGGT & 55 & X86782 \\
\hline crtOR & TGCCGAGCGTGAAATTGTGAGG & & \\
\hline $\operatorname{act} \mathrm{F}$ & CGTGAATGCCAGCAGCCTCCA & 55 & Huang et al., 2006 \\
\hline act $\mathrm{R}$ & & 55 & \\
\hline
\end{tabular}


maximum crtR-B expression occurred on day 12 with 7.6fold and day 8 with 6.3-fold in the EBR 25 and 50 treatments, respectively (Figure 4E). In the EBR 25 treatment, the initial increased expression of $l y c$ occurred on day 1.5 with 2.1-fold and the highest expression of lyc occurred on day 12 with 6.5-fold, whereas the initial increased and maximum lyc transcriptional occurred on day 0.5 with 2.8-fold and day 12 with 9.3-fold in the EBR 50 treatment (Figure 4F).

The patterns of bkt expression were similar to that of $l y c$. The $b k t$ level reached its initial increased and highest value on day 1.5 and day 12 in the EBR 25 treatment, which were 2.1- and 3.1-fold compared to the controls, respectively, whereas the first peak of $b k t$ level was on day 1.5 (2.4-fold) and the highest bkt level was on day 12 (11.5-fold) in the EBR50 treatment (Figure 4G). In the EBR 25 treatment, the initial increased and maximum transcriptional levels of crtO occurred on day 0.5 and 12, which were 2 -fold and 12-fold compared to the controls, respectively. In the EBR 50 treatment, the initial increased and maximum transcriptional levels of crtO occurred on day 0.5 (5.8-fold) and 4 (9.5-fold), respectively (Figure $4 \mathrm{H}$ ).

The results showed that the eight carotenogenic genes were up-regulated by EBR with different expression profiles. Moreover, EBR 25 induction had a greater effect on the transcriptional expression of ipi-1, ipi-2, crtR-B, lyc and $c r t \mathrm{O}$ (> 5- fold up-regulation) than on $p s y, p d s, b k t$ and EBR 50 treatment had a greater impact on the transcriptional expression of ipi-2, pds, lyc, crtR-B, bkt and crtO than on ipi-1 and psy.
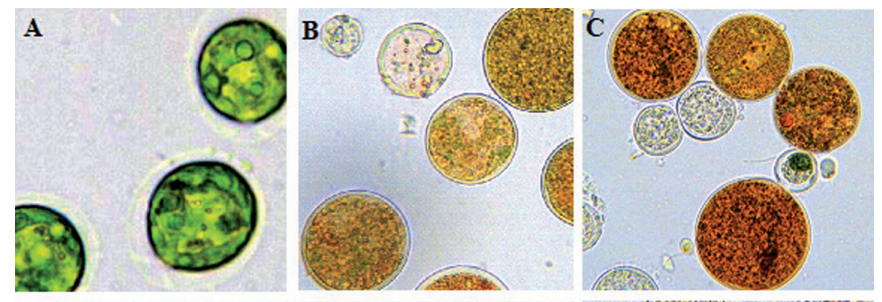

D
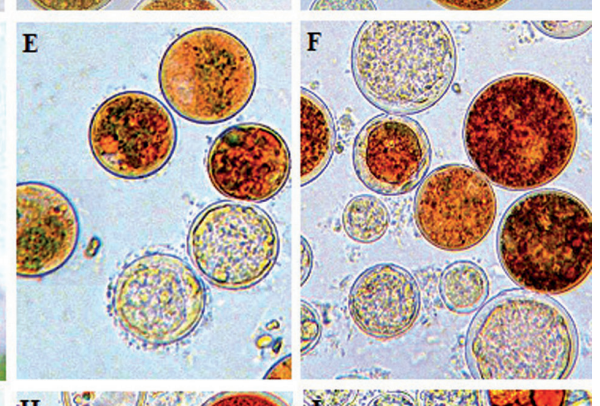

G
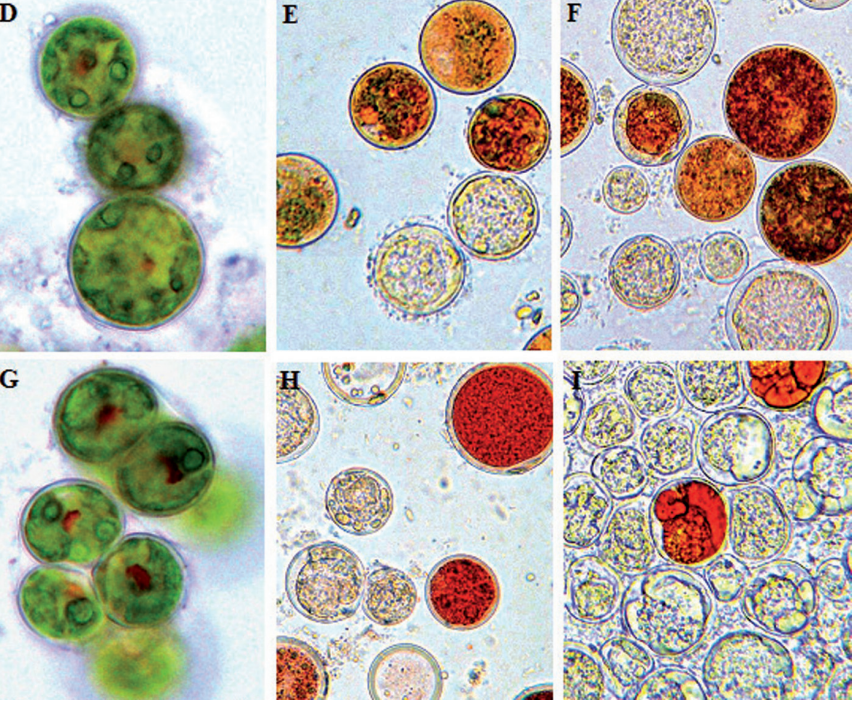

Figure 2: Microscopic images (400x) of $H$. pluvialis cells culture samples day 6,15 and 22 after treatments with EBR. A, B, C represent controls and treatments on day $6 ; D, E, F$ represent controls and treatments on day $15 ; \mathrm{G}, \mathrm{H}$, I represent controls and treatments on day 22 , respectively.

\section{DISCUSSION}

BRs play critical roles in a variety of physiological responses in the amelioration of various abiotic and biotic stresses in plants (Bajguz and Hayat 2009; Bajguz 2011). Bajguz (2010) reported that they played an important role in plant response to heavy metal stress and have an anti-stress effect on $C$. vulgaris contaminated by heavy metals. Dhaubhadel et al., (1999) demonstrated that after exposure to high-temperature stress, the EBR-treated seedlings of Brassica napus and tomato seedlings accumulated higher levels of four major classes of HSP (Heat Shock Protein), which may have a role in EBRmediated resistance to heat stress. Singh and Shono (2005) found that tomato plants treated with 24-epibrassinolide (EBR) were more tolerant to high temperature than untreated plants. Saygideger and Deniz (2008) demonstrated that EBR can reduce the impact of salinity stress on the growth of Spirulina platensis.

Carotenoids act as accessory light-harvesting pigments, and they perform an essential photoprotective role by quenching triplet state chlorophyll molecules and scavenging toxic oxygen radicals formed within the chloroplast (Young 1991). Astaxanthin plays a protective role under stress in $H$. pluvialis (Lemoine and Schoefs 2010). Therefore, it was thought that astaxanthin accumulating effectively under stress is a defensive reaction to stress and that astaxanthin plays a key role to neutralize stresses (Gao et al., 2012a). Results from our previous papers demonstrated that the accumulation of astaxanthin was directly related to the augmented photoprotection ability of $H$. pluvialis and higher level of astaxanthin accumulation under some kinds of stress-related phytohormones such as JA and SA, which might up-regulate mRNA expression of the eight carotenogenic genes effectively (Gao et al., 2012a, 2012b). It is unknown how BRs up-regulate the expression of carotenogenic genes and increase astaxanthin accumulation to enhance the ability to resist stresses of $H$. pluvialis.

BRs play key roles in plants under stresses (Bajguz and Hayat 2009; Bajguz, 2011) and astaxanthin accumulation is regarded as a defensive reaction to stress, which led us assess the relationship between these two facts. It was conjectured

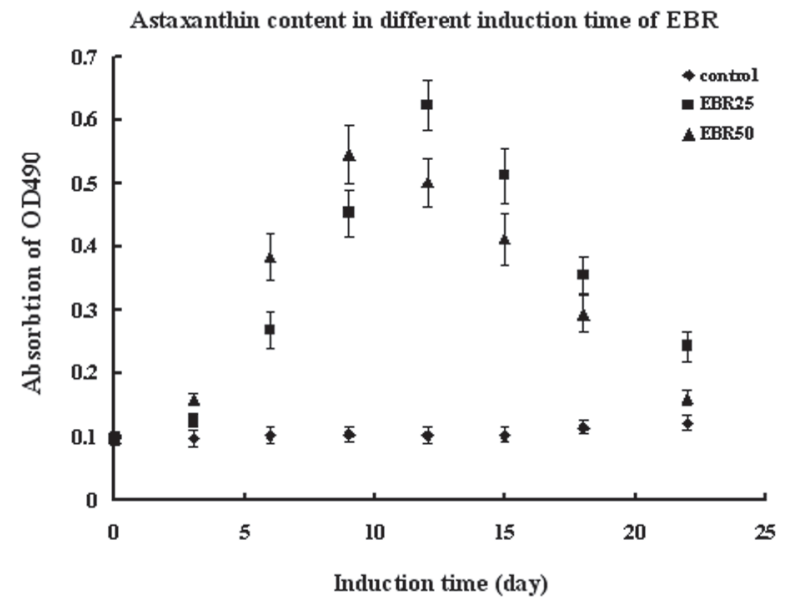

Figure 3: Astaxanthin accumulations of controls, EBR 25 samples and $\mathrm{EBR} 50$ samples during cultivation. $\mathrm{OD}_{490}$ represents relative astaxanthin content in alga culture solution. 
that exogenous EBR as an elicitor would enhance the capability of stress resistance by stimulating astaxanthin accumulation efficiently in $H$. pluvialis. In the present study, both EBR treatments were found to enhance astaxanthin production efficiently. However, the two different concentrations produced different astaxanthin acclamation profiles in $H$. pluvialis, which indicates the dual character of the induction. On one hand, EBR50 was more efficient to induce H. pluvialis to synthesize astaxanthin than EBR25 in the former part of induction course. On the other hand, EBR50 also resulted in more dead and bleached cells than EBR25 after application of the phytohormone for 12 days. At the same time, results of microscopic observation indicated that there were also serious side-effects of exposure to EBR in $H$. pluvialis. It led most cells to lose pigments and to bleach into shock at the end of cultivation course due to the higher concentration of EBR, which implies that 25 and 50mg/L EBR might be too high and be harmful to cell growth in $H$. pluvialis. Nevertheless, our results suggest that the algal solution should be harvested at the right time when using EBR as a biotic elicitor to produce astaxanthin on a large scale.

Fast astaxanthin accumulation was detected from the 3rd day in both EBR treatments in $H$. pluvialis, based on
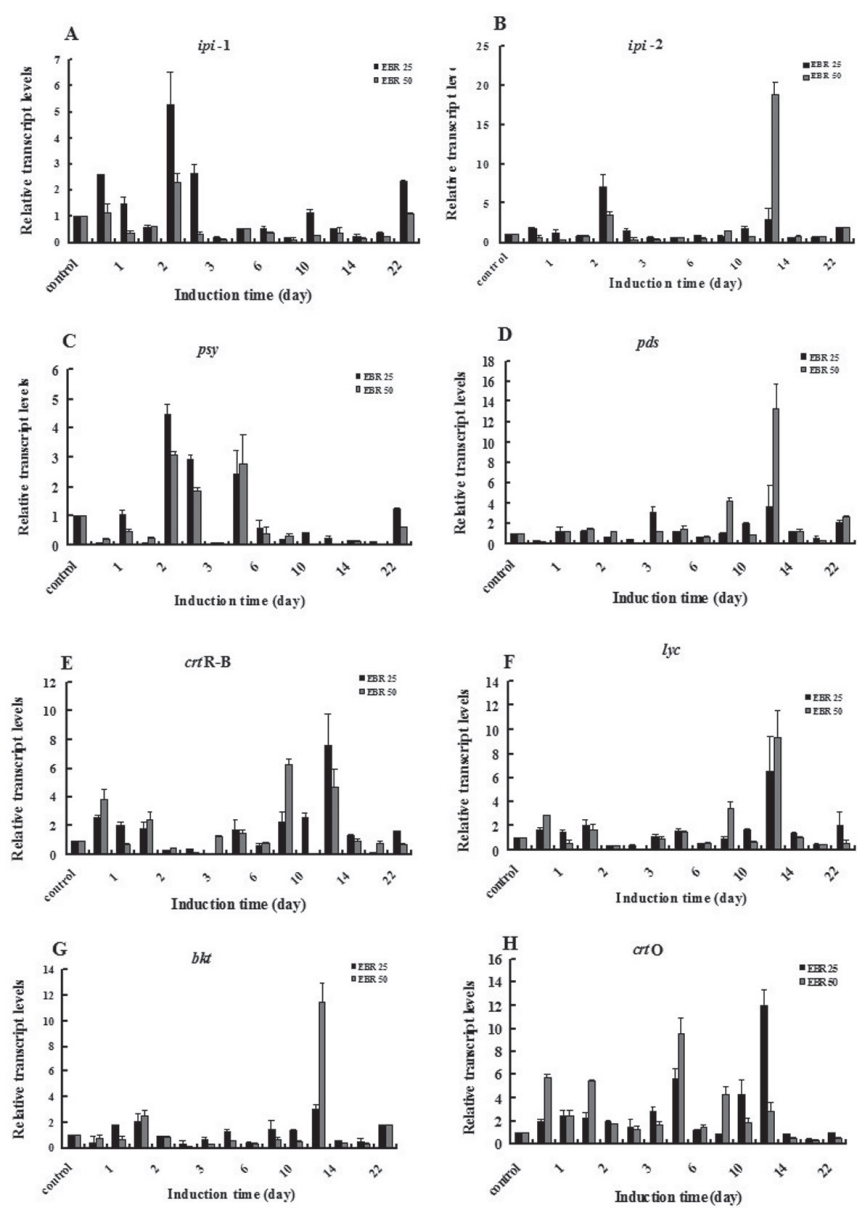

Figure 4: The effects of EBR on the transcript level expression kinetics of eight carotenogenic genes in $H$. pluvialis during incubation. $A, B, C, D, E, F, G$ and $H$ represent transcript levels expression kinetics of ipi-1, ipi-2, psy, pds, crtR-B, lyc, crtO and bkt, respectively. results of Figure 1 and Figure 2 in this paper. According to the correlation between transcriptional peaks of eight carotenogenic genes and the initiation time of fast astaxanthin accumulation, it can be inferred that they up-regulated astaxanthin accumulation at the transcriptional level, posttranscriptional level, or both levels (Li et al., 2008; 2010; Gao et al., 2012a; 2012b). The maximum transcriptional level of ipi-1, ipi-2 and psy appeared on day 2 in EBR 25 treatment, which preceded the time of initial fast accumulation of astaxanthin and indicates that the three genes might up-regulate astaxanthin biosynthesis at the post-transcriptional level in $H$. pluvialis. The other five genes, $p d s, l y c, c r t \mathrm{R}-\mathrm{B}, b k t$ and $c r \mathrm{O}$ expressed transcriptional peaks on day 12, which lagged behind the time point of fast accumulation of astaxanthin, thus the five genes might up-regulate astaxanthin biosynthesis in H. pluvialis at the transcriptional level. However, there were different changes in transcriptional expressions of the eight genes in the EBR 50 treatment. The maximum transcriptional peaks of $i p i-2, p d s, l y c, c r t \mathrm{R}-\mathrm{B}, b k t$ and $c r t \mathrm{O}$ appeared on day 12 , $12,12,8,12$ and 4, respectively, therefore these six genes might up-regulate astaxanthin biosynthesis at the transcriptional level. Both the mRNA expression maximum levels of ipi-1 and psy occurred on day 2, thus the two genes might up-regulate astaxanthin biosynthesis at the post-transcriptional level.

In conclusion, both ipi-1 and psy performed up-regulating astaxanthin biosynthesis at the post-transcriptional level, and $p d s, l y c, c r t \mathrm{R}-\mathrm{B}, b k t$ and $c r \mathrm{tO}$ acted at the transcriptional level in H. pluvialis, while ipi-2 up-regulated biosynthesis at the post-transcriptional level in EBR 25 and at the transcriptional level in the BR50 treatment. It it suggested that ipi-2 might control astaxanthin biosynthesis at both the transcriptional and post-transcriptional levels. These results were different from our previous results with JA and SA, the other stress-related phytohormones. Astaxanthin biosynthesis in the presence of JA appeared to be up-regulated mainly by $p s y, p d s, c r t \mathrm{R}-\mathrm{B}, l y c, b k t$ and $c r t \mathrm{O}$ at the transcriptional level and ipi-1 and ipi-2 at both transcriptional and post-transcriptional levels in $H$. pluvialis (Gao et al., 2012a). SA induced astaxanthin accumulation by up-regulating ipi-1, ipi-2, psy, crtR-B, bkt and $c r t \mathrm{O}$ at the transcriptional level and $l y c$ at the post-transcriptional level and $p d s$ at both levels H. pluvialis (Gao et al., 2012b). These results indicate that the three stress-related phytohormones could induce $H$. pluvialis accumulate astaxanthin efficiently; however, they might have different regulatory profiles.

\section{ACKNOWLEDGMENTS}

The present study was supported by the National Natural Science Foundation of China $(41106124,31170279)$, the National Natural Science Foundation of Shandong Province (ZR2011DM006, ZR2011CQ010), the open funds of the State Key Laboratory of Agricultural Microbiology (AMLKF201003) and the supporting project for young teachers in Shandong University of Technology.

\section{REFERENCES}

ANURADHA S, RO SSR (2001) Effect of brassinosteroids on salinity stress induced inhibition of seed germination and seedling growth of rice (Oryza sativa L.). Plant Growth Regul 33: 151-153.

ANURADHA S, RO SSR (2009) Effect of 2, 4-epibrassinolide on the photosynthetic activity of radish plants under cadmium stress. Photosynthetica 47: 317-320. 
BAJGUZ A, CZERPAK R (1996) Effect of Brassinosteroids on growth and proton extrusion in the Alga Chlorella vulgaris Beijerinck (Chlorophyceae). J Plant Growth Regul 15:153-156.

BAJGUZ A, CZERPAK R (1998) Physiological and biochemical role of Brassinosteroids and their structure-activity relationship in the green alga Chlorella vulgaris Beijerinck (Chlorophyceae). J Plant Growth Regul 17:131-139.

BAJGUZ A, HAYAT S (2009) Effects of brassinosteroids on the plant responses to environmental stresses. Plant Physiol Biochem 47:1-8.

BAJGUZ A (2010) An enhancing effect of exogenous brassinolide on the growth and antioxidant activity in Chlorella vulgaris cultures under heavy metals stress. Environ Exp Bot 68:175-179

BAJGUZ A (2011) Suppression of Chlorella vulgaris Growth by Cadmium, Lead, and Copper Stress and Its Restoration by Endogenous Brassinolide. Arch Environ Contam Toxicol 60:406-416

BOROWITZKA, MA, HUISMAN, JM, OSBORN, A (1991) Culture of the astaxanthin-producing green alga Haematococcus pluvialis. 1. Effects of nutrients on growth and cell type. J Appl Phyco 3: 295-304.

CARANGE J, LONGPRE F, DAOUST B, MARTINOLI MG (2011) 24-epibrassinolide, a phytosterol from the Brassinosteroid family, protects dopaminergic cells against MPP+-induced oxidative stress and apoptosis. J Toxicol Article ID 392859, 13 pagesdoi:10.1155/2011/392859.

CHOUDHARY SP, BHARDWAJ R, GUPTA BD, DUTT P, GUPTA RK, KANWAR M, BIONDI S (2011) Enhancing effects of 2,4-epibrassinolide and Putrescine on the antioxidant capacity and free radical scavenging activity of Raphanus satious seedlings under Cu ion stress. Acta Physiol Plant 33:1319.1333.

DALIO RJD, PINHEIRO HP, SODEK L HADDAD CRB (2011) The effect of 24-epibrassinolide and clotrimazole on the adaptation of Cajanus cajan (L.) Millsp. to salinity. Acta Physiol Plant 33:1887.1896.

DHAUBHADEL S CHAUDHARY S, DOBINSON KF, KRISHNA P (1999) Treatment with 2,4-epibrassinolide, a brassinosteroid, increases the basic thermotolerance of Brassica napus and tomato seedlings. Plant Mol Biol 40: 333.342.

DING J, SHI K and ZHOU YH (2009) Effects of root and foliar applications of 24-Epibrassinolide on fusarium wilt and antioxidant metabolism in Cucumber Roots. Hortscience 44:1340.1345.

GAO ZQ, MENG CX, YE NH, HU BW, LUO T, WANG YT (2011). A method of using 2,4 -epibrassinolide (EBR) to induce $H$. pluvialis produce astaxanthin quickly. Chinese Patent, NO. ZL200610171008X

GAO ZQ, MENG CX, ZHANG XW, XU D, MIAO XX, WANG YT, LV HX, YANG LM, CHEN LL, YE NH (2012a) Differential expression of carotenogenic genes, associated changes on astaxanthin production and photosynthesis features induced by JA in H. pluvialis. PLoS ONE 7: 1-7.

GAO ZQ, MENG CX, ZHANG XW, XU D, ZHAO YF, WANG YT, YANG LM, LV HX, CHEN LL, YE NH. (2012b) Induction of salicylic acid (SA) on transcriptional expression of eight carotenoid genes and astaxanthin accumulation in Haematococcus pluvialis. Enzyme Micro Technol 51: 225230.

GRÜNEWALD K, ECKERT M, HIRSCHBERG J, HAGEN C (2000) Phytoene desaturase is localized exclusively in the chloroplast and up-regulated at the mRNA level during accumulation of secondary carotenoids in Haematococcus pluvialis (Volvocales, Chlorophyceae). Plant Physiol 122: 1261-1268.

HOWELL WM, KELLER III GE, KIRKPATRICK JD, JENKINS RL, HUNSINGER RN, MCLAUGHLIN EW (2007) Effects of the plant steroidal hormone, 2, 4-Epibrassinolide, on the mitotic index and growth of onion (Allium cepa) root tips. Genet Mol Res 6: 50-58.

HUNG JC, CHEN F, SANDMAN G (2006) Stress-related differential expression of multiple b-carotene ketolase genes in the unicellular green alga Haematococcus pluvialis. J Biotechnol 122: 176-185.

KANG YY, GUO SR, LI J, DUAN JJ (2009) Effect of root applied 2,4-epibrassinolide on carbohydrate status and fermentative enzyme activities in cucumber (Cucumis sativus L.) seedlings under hypoxia. Plant Growth Regul 57: 259-269.
LEMOINE Y, SCHOEFS B (2010) Secondary ketocarotenoid astaxanthin biosynthesis in algae:a multifunctional response to stress. Photosyn Res 106: 155-177.

LI KR, WWANG HH, HAN G, WANG QJ, FAN J (2008) Effects of brassinolide on the survival, growth and drought resistance of Robinia pseudoacacia seedlings under water-stress. New Forests 35: 255-266

LI YT, SOMMERFELDA M, CHEN F, HU Q (2008) Consumption of oxygen by astaxanthin biosynthesis: A protective mechanism against oxidative stress in Haematococcus pluvialis (Chlorophyceae). J Plant Physiol 165: 1783-1797.

LI YT, SOMMERFELDA M, CHEN F, HU Q (2010) Effect of photon flux densities on regulation of carotenogenesis and cell viability of Haematococcus pluvialis (Chlorophyceae). J Appl Phycol 22: 253-263.

LI J, ZHU DL, NIU JF, SHEN SD, WANG GC (2011) An economic assessment of astaxanthin production by large scale cultivation of Haematococcus pluvialis. Biotechnol Adv, 29: 568-74

LI YH, LIU YJ, XU XL, JIN M, AN LZ, ZHANG H (2012) Effect of 2,4-epibrassinolide on drought stress-induced changes in Chorispora bungeana. Biol Plant 56: 192-196

LIVAK KJ, SCHMITTGEN TD (2001) Analysis of relative gene expression data using real-time quantitative PCR and the $2-\Delta \Delta C T$ method. Methods 25: 402-408.

OZDEMIR F, BOR M, DDEMIRAL T, TURKAN I (2004) Effects of 24-epibrassinolide on seed germination, seedling growth, lipid peroxidation, proline content and antioxidative system of rice (Oryza sativa L.) under salinity stress. Plant Growth Regul 42: 203-211.

PARK WJ (1998) Effect of epibrassinolide on hypocotyl growth of the tomato mutant diageotropica. Planta 207: 120-124.

PUSTOVOITOVA TN, ZHDANOVA NE, ZHOLKEYICK VN (2001) Epibrassinolide increases plant drought resistance. Doklady Biochem Biophys 376: 36-38.

SAMIRA IMH, DRIDI-MOUHANDES B, MANSOUR-GUEDDES S, DENDEN M (2012) 24-Epibrassinolide ameliorates the adverse effect of salt stress $(\mathrm{NaCl})$ on pepper (Capsicum annuum L.) J Stress Physiol Biochem, 8: 232-240.

SAYGIGERDER S, DENZ F. (2008) Effect of 2,4-epibrassinolide on biomass, growth and free proline concentration in Spirulina platensis (Cyanophyta) under $\mathrm{NaCl}$ stress. Plant Growth Regul 56: 219-223.

SHAHID MA, PERVEZMA, BALAL RM, MATTSON NS, RASHID A, AHAMD R, AYYUB CM, ABBAS T (2011) Brassinosteroid (24-epibrassinolide) enhances growth and alleviates the deleterious effects induced by salt stress in pea (Pisum sativum L.) AJCS 5: 500-510.

SHARMA P, BHARDWAJ R (2007) Effect of 2,4-epibrassinolide on seed germination, seeding growth and heavy metal uptake in Brassica Junceal. Gen Appl plant physiol 33: 59-73.

SHARMA P, BHARDWAJ R (2007) Effects of 2,4-epibrassinolide on growth and metal uptake in Brassica juncea L. under copper metal stress. Acta Physiol Plant 29: 259-263.

SHARMA I, PATI PK, BHARDWAJ R (2011) Effect of 24-epibrassinolide on oxidative stress markers induced by nickel-ion in Raphanus sativus L. Acta Physiol Plant 33: 1723-1735

SINGH I, SHONO M. (2005) Physiological and molecular effects of 2,4-epibrassinolide, a brassinosteroid on thermotolerance of tomato. Plant Growth Regul 47: 111-119.

UPRET KK, MURT GSR (2004) Effects of brassinosteroids on growth, nodulation, phytohormone content and nitrogenase activity in French bean under water stress. Biol Plant 48: 407-411.

YOKOTA T, KIM SK, FUKUI Y, TAKAHASHI N, TAKEUCHI Y, TAKEMATSU T (1987) Brassinosteroids and sterols from a green alga, Hydrodictyon reticulatum: Configuration at C-24. Phytochemistry 26: 503-506.

YOUNG AJ (1991) The photoprotective role of carotenoids in higher plants. Physiol Plantar 83: 702-708. 